\title{
Assessment of Cognitive Abilities in Preschool Children with and without Developmental Coordination Disorder
}

\author{
${ }^{1}$ Katerina Asonitou, ${ }^{2}$ George Tsiganos, ${ }^{1}$ Thomas Kourtessis, ${ }^{1}$ Georgia Strofylla, ${ }^{1}$ Dimitra \\ Koutsouki \\ ${ }^{1}$ Laboratory of Adapted Physical Activity/Developmental and Physical Disabilities \\ Department of Physical Education \& Sports Science, National \& Kapodistrian University of \\ Athens, Greece \\ ${ }^{2}$ Department of Physical Education \& Sport Science, Democritus University of Thrace, \\ University Campus, 69100 Komotini, Greece
}

\begin{abstract}
Differences in cognitive abilities between 54 Greek children with developmental coordination disorder (DCD) and 54 children without DCD, 5-and 6-yearold, were evaluated on the three subscales of the Cognitive Assessment System (CAS: Naglieri \& Das, 1997a). Children with DCD performed significantly lower than the control group on the Planning, Attention and Simultaneous Processing Scales of the standardized CAS. In discriminative function analysis the tasks of the "Planning scale" and the "Figure Memory task" of the Simultaneous scale were the best discriminators between the two groups. The data were analysed using SPSS 19.0 for Windows (Norusis, 2011). In the current study, the cognitive performance of students with DCD was inferior to the control group in all cognitive tasks. The results suggest that children with DCD with significant difficulties in planning, spatial organization and memory mechanisms may probably be at risk for academic failure in the future. Early detection of cognitive-motor difficulties may be essential for early intervention.
\end{abstract}

\section{Introduction}

Developmental Coordination Disorder (DCD) is characterized by children's difficulties in acquisition and execution of motor skills [10]. This disorder in motor coordination is not caused by a pathologicalneurological or mental problem and it is intertwined with learning environment resulting, in more cases, in academic failure [18]. According to DSM-IV [1] the incidence of the disorder is $6-10 \%$ of ages $5-11$, but the percentages vary among researchers.

Previous studies revealed a cognitive dysfunction profile of children with DCD attributing clumsiness to impaired information processing system (visualperceptual, visual-spatial processing disorders), planning, and memory and learning difficulties [17].

Getchell and his colleagues [6] examined the motor coordination in children with and without learning disabilities (mean: 8.6 years old). Their study showed that motor coordination skills provided important information for early detection of learning difficulties, as a novelty. The researchers suggested the importance of the theoretical approach of 'dynamical systems' (interaction perceptual, cognitive and motor capacity). Also, motor coordination can be a "window" for understanding the mechanisms in learning disabilities [6].

For the purpose of the current study the PASS theory was adopted (Planning, Attention, Simultaneous and Successive Coding) and the normreferenced Cognitive Assessment System (CAS) [14] was used to assess the children's cognitive abilities. According to this theory the following four interrelated components are responsible for human action. Attention is the ability to concentrate on a stimulus in order to process the incoming information. Alertness and attention are essential prerequisites for effective coding and planning [5]. When the stimulus is received by perception then people analyze, synthesize and arrange information according to the two types, Simultaneous and Successive Coding. Simultaneous coding arrange information in a single group (e.g. cat, dog, bird are all pets), while Successive coding arrange information in a sequential order (e.g. the digits in a telephone number). Planning ability helps people to make plans in order to respond and act in the incoming information and then to judge and evaluate their reaction. Each motor and/or cognitive activity is designed and built through the involvement of the cognitive functions and knowledge base. The knowledge base is the accumulated storage of knowledge, which a person has acquired from previous experiences [20]. CAS implements the PASS theory. 


\section{Method}

Differences in cognitive abilities between 54 Greek preschoolers with DCD and 54 without DCD, 5-and 6-year-old, were assessed on three CAS Scales, using PASS theory. All students were enrolled in a kindergarten school and were never diagnosed with neurological and emotional disorders, physical disabilities, autistic disorders or intellectual disabilities. Because of the clear evidence of the co morbid nature of DCD (e.g. 30-35\% of children who have ADHD also have DCD) [19] the CAS Attention Scale was used in line with DSM-IV criteria.

The total participants were consisted of 108 students (71 boys and 37 girls) (Mean age $=69.02$ months, $S D=3.72$, Minimum $=60$ months, Maximum=77 months). The (age band 1: ages 4-6) norm-referenced test Movement Assessment Battery for Children (MABC) [8] was used to assess motor difficulties and confirm the existence or none of DCD.

The total time of administration ranged from 20 to $40 \mathrm{~min}$. The MABC assess performance in three motor domains: a) manual dexterity, b) ball skills, and c) static and dynamic balance.

The normative Total Impairment Score (TIS) (from " 0 " to "40") was the selection criterion of differentiating children with and without DCD. Children who had a TIS between the $15^{\text {th }}$ and $6^{\text {th }}$ percentiles were characterized as "at risk" with moderate difficulties, and children who had a TIS below the $5^{\text {th }}$ percentile were characterized as "severe motor problems". Reliability and validity of the MABC are satisfying and available in the test's Manual [8].

All students were assessed with the MABC and fifty four of them (35 boys and 19 girls) were found as having DCD. According to the MABC norms, 54 children had a total motor score from " 0 to 9.5 " (above the $15^{\text {th }}$ percentile) and were characterized as "non DCD", 15 children had a total impairment score (TIS) from "10 to 14 " (between the $15^{\text {th }}$ and $6^{\text {th }}$ percentiles) and were characterized as "at risk" with moderate difficulties, and 39 children had a total impairment score (TIS) from "15 to 34 " (below the $5^{\text {th }}$ percentile) and were characterized as "severe motor problems".

The control group consisted of 54 children (35 boys and 19 girls) of the same age and gender with the children of the experimental group. All students were enrolled in a kindergarten school and were never diagnosed with neurological and emotional disorders, physical disabilities, autistic disorders or intellectual disabilities.
For the purpose of the present study the three cognitive scales a) Planning Scale (3 subtests), b) Simultaneous Coding Scale (3 subtests), and c) Attention Scale (3 subtests) of the standard battery of CAS were used. CAS give us a cognitive profile about the strengths and weaknesses of the children's intellectual abilities and how they process the incoming information.The standard scores of the scales are relevant to successes and failures in specific academic areas and predict academic performance [13]. The cognitive abilities meet specific academic areas (reading, spelling, mathematics, reading comprehension) $[9 ; 11 ; 15]$. Lower standard scores indicate probable learning disabilities. Specifically, scores below 90 (the sum of the individual tasks) for the Planning, Attention, and Simultaneous Information Coding identify the possible presence of learning difficulties related to the scale. Reliability and validity of the CAS are satisfying and available in the test's Manual [13].

The CAS allows for early detection of cognitive disorders in preschool children, which lead to learning difficulties during primary school. Besides the detection and assessment of learning difficulties, the CAS can be applied to children with mental retardation syndrome, attention deficit hyperactivity, severe emotional disorders, traumatic brain injury, as well as gifted students [13].

\section{Analysis and Results}

The data were analyzed using SPSS 19.0 for Windows [16]. A probability level of $\mathrm{p}<.05$ was considered statistically significant.

The performance differences of DCD and non DCD children on nine cognitive tasks were analyzed using the multivariate analysis of variance (MANOVA) [16]. "Planning", "Simultaneous Coding" and "Attention" scales were considered as depended variables, while "groups" (DCD/ non DCD children) was the independent factors.

Multivariate analyses of significance were used to test the effects of each factor and all possible interactions. These effects were evaluated through univariate F-tests of significance on each dependent variable.

Descriptive discriminant function analyses were utilized as the post hoc tests to determine significant effects. It was also used to assess which of the subscale scores were significant predictors in classifying participants as DCD or non DCD children. Eta squared was used as the measure of effect size.

MANOVA showed important differences between the two groups in the area of "Planning" (see Table 1). The descriptive discriminant analysis indicated that 
the "Planned Codes" and "Planned Connections" tasks of Planning scale significantly separated DCD and non DCD children. The percentage of children correctly classified into DCD and non DCD groups was $80.8 \%$ overall. All indicators were significant (see Table 2).

Table 1. MANOVA comparing DCD and non DCD groups on Planning Scale

\begin{tabular}{|c|c|c|c|c|c|c|}
\hline 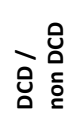 & ధุ & \begin{tabular}{l}
$\stackrel{0}{N}$ \\
\multirow{A}{*}{}
\end{tabular} & 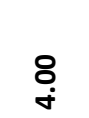 & $\begin{array}{l}8 \\
\text { த் }\end{array}$ & ઠ̊ & 马े \\
\hline 莺 & 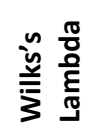 & 4 & 这 & 京 & e & 莣 \\
\hline
\end{tabular}

Table 2. Descriptive Discriminant Function Analysis on Planning Scale's three subtests

\begin{tabular}{|c|c|c|c|c|c|}
\hline \multicolumn{6}{|c|}{$\mathbf{8 2 . 7 \%}$ of original grouped cases correctly classified } \\
\hline 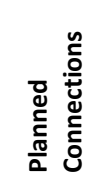 & ఫ̊ & ઠे & 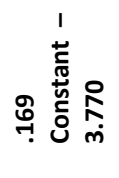 & ڤัర & Џ్ \\
\hline 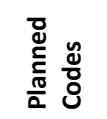 & 웅 & ષิ & $\underset{\sim}{\mathbb{J}}$ & ర̊ & $\underset{\infty}{\stackrel{R}{\infty}}$ \\
\hline 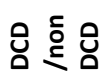 & & & & & \\
\hline $\begin{array}{l}\frac{0}{0} \\
\frac{0}{\frac{\pi}{2}} \\
\frac{50}{7}\end{array}$ & 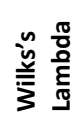 & e & 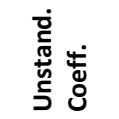 & 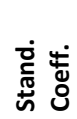 & 蓠 \\
\hline
\end{tabular}

Also, MANOVA showed important differences between the two groups in the area of "Simultaneous Processing/Coding" of information (see Table 3). The descriptive discriminant analysis indicated that the "Figure Memory" and "Verbal Spatial Relations" tasks of Simultaneous scale significantly separated
DCD and non DCD children. The percentage of children correctly classified into DCD and non DCD groups was $78.8 \%$ overall. All indicators were significant (see Table 4).

Table 3. MANOVA comparing DCD and non DCD groups on Simultaneous Scale

\begin{tabular}{|c|c|c|c|c|c|c|}
\hline 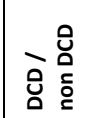 & :̣̊̂ & 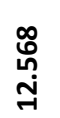 & ه্ & $\begin{array}{l}8 \\
\infty \\
\infty\end{array}$ & ঃั & गี \\
\hline 䓌 & 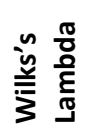 & 4 & $\stackrel{\circ}{\grave{\lambda}}$ & 袬 & 2 & 焉 \\
\hline
\end{tabular}

Table 4. Descriptive Discriminant Function Analysis on Simultaneous Scale's three subtests

\begin{tabular}{|c|c|c|c|c|c|}
\hline \multicolumn{6}{|c|}{$78.8 \%$ of original grouped cases correctly classified } \\
\hline 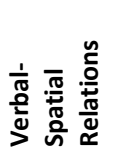 & 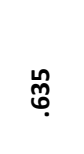 & ఫุ & 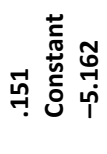 & $\underset{n}{\stackrel{N}{m}}$ & Y̛ \\
\hline 产 & بْ & ఫ̊ & 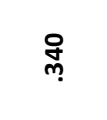 & $\underset{\infty}{\stackrel{-}{\mid}}$ & ڤ̆ \\
\hline 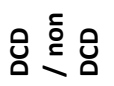 & & & & & \\
\hline$\frac{0}{\frac{0}{0}}$ & 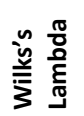 & 2 & 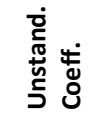 & 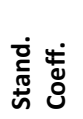 & 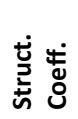 \\
\hline
\end{tabular}

Regarding “Attention” scale MANOVA showed important differences between the two groups (see Table 5). The descriptive discriminant analysis indicated that the "Number Detection" and "Expressive Attention" tasks of Attention scale significantly separated DCD and non DCD children. The percentage of children correctly classified into DCD and non DCD groups was $64.4 \%$ overall. All indicators were significant (see Table 6). 
Table 5. MANOVA comparing dcd and non ded groups on Attention Scale

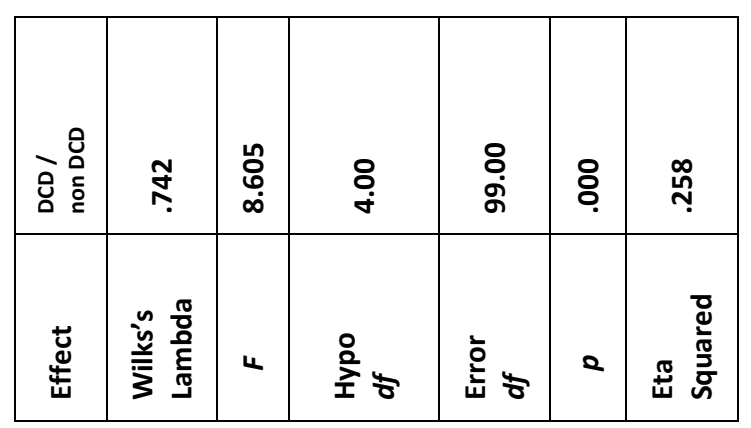

Table 6. Descriptive Discriminant Function Analysis on Attention Scale's three subtests

\begin{tabular}{|c|c|c|c|c|c|}
\hline \multicolumn{6}{|c|}{$\begin{array}{l}\text { 64.4\% of original grouped cases correctly } \\
\text { classified }\end{array}$} \\
\hline 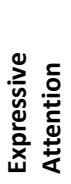 & 華 & চి & 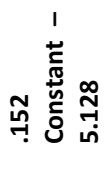 & \&্ & ํㅗํ \\
\hline ס & $\stackrel{-1}{\stackrel{-1}{\uparrow}}$ & চ̊ & $\stackrel{\text { ตे }}{\text { m. }}$ & 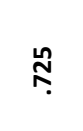 & ণุ \\
\hline 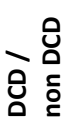 & & & & & \\
\hline $\begin{array}{l}\frac{0}{0} \\
\frac{0}{\frac{\pi}{5}} \\
\frac{10}{>}\end{array}$ & 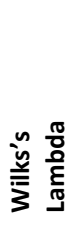 & e & 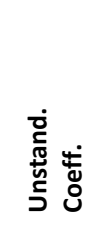 & 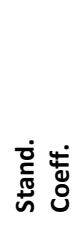 & 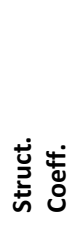 \\
\hline
\end{tabular}

Only the "Planned Codes" and the "Figure Memory" tasks (of the total nine cognitive tasks) in the discriminant function analysis differentiated the groups. The structure coefficient of .82 for "Planned Codes" and .76 for "Figure Memory" tasks were reflective of the mean for DCD children being lower than the mean of non DCD children. The percentage of children correctly classified into DCD and non DCD groups was $83.2 \%$ overall. All indicators were significant (for the "Planned Codes" tasks: Wilk's $\Lambda=$ $.671, \mathrm{p}=.000$ and for the "Figure Memory" tasks: Wilk's $\Lambda=.581, \mathrm{p}=.000)$.

\section{Discussion}

Children with DCD performed significantly lower than the control group on the "Planning", "Attention" and "Simultaneous Processing" Scales. Further analyses showed that "Planned Codes" and "Planned Connections" subtests of Planning scale significantly separated DCD and non DCD children in percentage $80.8 \%$. This percentage was the biggest than the others subtests of the two Scales (Simultaneous and Attention) correctly classified children into two groups. Further analyses showed important differences between the two groups, especially in the "Figure Memory" and "Verbal Spatial Relations" subtests of Simultaneous scale, "Number Detection" and "Expressive Attention" subtests of Attention scale. These subtests significantly separated DCD and non DCD children. When discriminant function analysis performed on total nine cognitive tasks "Planned Codes" and "Figure Memory" standard subtests scores were the only significant discriminators between the two groups.

The results in the current study demonstrated clearly deficits in planning and decision-making processes. Also, the findings showed difficulties in spatial organization mechanisms with greater difficulty in "figure memory" among the children with DCD. The results are in consistency with previous study, where lower levels of cognitive performance were found on three CAS scales among children with DCD [2]. Also in the previous findings, cognitive subtests as matching numbers1, planning codes1, non-verbal matrices, figure memory and number detection 2 aappeared to be the best discriminators between the two groups [3].

The results of the present study provide support for previous findings on planning and decisionmaking processing [12] as well as visuo-spatial processing deficits [18]. Improper or inadequate evaluation leads children with DCD to improper or inadequate planning, which in turn lead to failure of the project. Martini et al. [12] suggested the use of research strategies to understand the decisionmaking processes during different motor tasks. Rosenblum and Livneh-Zirinski [18] found that both handwriting process (name writing and the paragraph copying tasks) provide a more comprehensive picture of the DCD children. Such deficits may influence academic success indicating possible relationships between coordination problems, handwriting deficits, dyslexia and literacy acquisition problems, which may lead in emotional and social dysfunction. 
In the current study the characteristically low performance in the "Figure Memory" subtest is a product characteristic of the handwriting process for the children with DCD.

Children with DCD often appear to have a narrow repertoire of cognitive strategies with organizational, planning, memory and learning difficulties [17]. The implications of cognitive skills in different mathematical tasks, and in relation to the impact of limited cognitive skills in the classroom environment have been studied from researchers. Bull, Espy and Wiebe [4] examined whether measures of short-term memory, working memory, and executive functioning in preschool children predict later proficiency in academic achievement at 7 years of age (third year of primary school). They revealed that visual short-term and working memory may predict math achievement, while executive function skills predicted learning in general rather than learning in one specific domain. In another study, Planning and Simultaneous processing abilities are important predictors and most related to early math skills [11].

Planning processes are required for making decisions about how to solve a math problem, recall and apply certain math facts, monitor and evaluate one's performance. Simultaneous processes are required for the solution of math problems, as they consist of different elements which students must be integrated into a whole in order to answer [11]. Also, poor performance on Planning and Simultaneous coding has been confirmed as explanatory of reading comprehension difficulties $[7 ; 15]$.

In the current study early identification of strengths and weaknesses of preschool children with and without DCD was evaluated via PASS theory and CAS. Revealing cognitive deficits in preschool children with DCD we may be able to predict academic problems because of the strong relationship between CAS achievement tasks and PASS processes [15]. The "Planning" Scale and "Figure Memory" task meet specific academic areas (reading, spelling, mathematics, reading comprehension) $[9 ; 11 ; 15]$.

The implications for using PASS theory to assist in the identification process as well as for the design of interventions for children with Attention Deficit/ Hyperactivity Disorder (ADHD) have been reported in the past as well [15].

\section{Conclusion}

The results suggest that preschoolers with DCD may probably be at risk for academic failure in the future. Early identification of cognitive-motor difficulties may be essential for early intervention in order to anticipate and improve future academic and motor difficulties. We could propose a cognitivemotor based approach to enhance cognitive as well as motor performance of children with DCD.

\section{References}

[1] American Psychiatric Association (APA). (1994), 'Diagnostic and statistical manual of mental disorders $\left(4^{\text {th }}\right.$ ed.)', p. 37-55, Washington, DC: Author.

[2] Asonitou, K., Koutsouki, D., Kourtessis, T., \& Charitou, S. (2012). Motor and cognitive performance differences between children with and without developmental coordination disorder (DCD). Research in Developmental Disabilities, 33, 996-1005.

[3] Asonitou, K., Koutsouki, D., \& Charitou (2010). Motor skills and cognitive abilities as a precursor of academic performance in children with and without DCD. Procedia Social and Behavioral Sciences, 5, 1702-1707.

[4] Bull, R., Espy, K., \& Wiebe, S. (2008) Short-Term memory, working memory and executive functioning in preschoolers: longitudinal predictors of mathematical achievement at age 7 years. Developmental Neuropsychology, 33, 205-228.

[5] Das, J. P. (1986), 'Information processing and motivation as determinants of performance in children with learning disabilities', In H. Whiting, \& M. Wade (Eds.), 'Themes in motor development', 127-142. Hingham, MA: Kluwer-Academic.

[6] Getchell, N., McMenamin, S., \& Whitall, J. (2005). Dual motor task coordination in children with and without learning disabilities. Adapted Physical Activity Quarterly, 22, 21-38.

[7] Haddad, F., Garcia, Y., Naglieri, J., Grimditch, M., McAndrews, A., \& Eubanks, J. (2003). Planning facilitation and reading comprehension: instructional relevance of the Pass theory. Journal of Psychoeducational Assessment, 21, 282-289.

[8] Henderson, S. E., \& Sugden, D. A. (1992), 'Movement assessment battery for children', London: The Psychological Corporation Ltd.

[9] Huang, L., Bardos, A., \& Carl D'Amato, R. (2010), 'Identifying students with learning disabilities: composite profile analysis using the Cognitive Assessment System', Journal of Psychoeducational Assessment, vol. 28, p. 19-30.

[10] Koutsouki, D. (2001), 'Special Physical Education: theory and practice', Athens, Greece: Athlotypo. Available in: www.athlotypo.gr.

[11] Kroesbergen, E., Van Luit, J., Naglieri, J., Taddei, S., \& Franchi, E. (2010), 'PASS Processes and Early Mathematics 
Skills in Dutch and Italian Kindergarteners', Journal of Psychoeducational Assessment, vol. 28, p. 585-593.

[12] Martini, R., Wall, A. E. T., \& Bruce, M. S. (2004). Metacognitive processes underlying psychomotor performance in children with differing psychomotor abilities. Adapted Physical Activity Quarterly, 21, 248-268.

[13] Naglieri J. A., \& Das, J. P. (1997a), 'Cognitive Assessment System. Itasca’, IL: Reverside Publising.

[14] Naglieri J. A., \& Das, J. P. (1997b), 'Cognitive Assessment System administration and scoring manual', Itasca, IL: Reverside Publising.

[15] Naglieri, J., Salter, C., \& Edwards, G. (2004), 'Assessment of children with attention and reading difficulties using the Pass theory and cognitive assessment system', Journal of Psychoeducational Assesssment, vol. 22, p. 93-105.

[16] Norusis, M. (2011), 'IBM SPSS Statitistics 19 Guide to data analysis', Pearson. UK: SPSS Ink.

[17] Ricon, T. (2010), 'Using concept maps in cognitive treatment for children with developmental coordination disorder', Health, vol. 2, p. 685-691.

[18] Rosenblum, S., \& Livneh-Zirinski, M. (2008), 'Handwriting process and product characteristics of children diagnosed with developmental coordination disorder', Human Movement Science, vol. 27, p. 200-214.

[19] Salmon, G. \& Kirby, A. (2008) Schools: Central to Providing Comprehensive CAMH Services in the Future? Child and Adolescent Mental Health, 13 (3), 107-114.

[20] Wall, A. E. T. (2004), 'the developmental skill-learning gap hypothesis: Implications for children with movement difficulties', Adapted Physical Activity Quarterly, vol. 21, p. 197-218. 